\title{
Comprehensive bioinformatics analysis of critical IncRNAs, mRNAs and miRNAs in non-alcoholic fatty liver disease
}

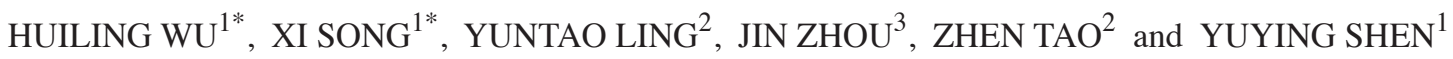 \\ Departments of ${ }^{1}$ General Practice, ${ }^{2}$ Infectious Diseases and ${ }^{3}$ General Surgery, Nanjing First Hospital, \\ Nanjing Medical University, Nanjing, Jiangsu 210006, P.R. China
}

Received March 24, 2018; Accepted December 10, 2018

DOI: $10.3892 / \mathrm{mmr} .2019 .9931$

\begin{abstract}
Non-alcoholic fatty liver disease (NAFLD) is the most common fatty liver disease in developed countries, in which fat accumulation in the liver is induced by non-alcoholic factors. The present study was conducted to identify NAFLD-associated long non-coding RNAs (lncRNAs), mRNAs and microRNAs (miRNAs). The microarray dataset GSE72756, which included 5 NAFLD liver tissues and 5 controls, was acquired from the Gene Expression Omnibus database. Differentially expressed lncRNAs (DE-lncRNAs) and mRNAs (DE-mRNAs) were detected using the pheatmap package. Using the clusterProfiler package and Cytoscape software, enrichment and protein-protein interaction (PPI) network analyses were conducted to evaluate the DE-mRNAs. Next, the miRNA-lncRNA-mRNA interaction network was visualized using Cytoscape software. Additionally, RP11-279F6.1 and AC004540.4 expression levels were analyzed by reverse transcription quantitative polymerase chain reaction. There were 318 DE-lncRNAs and 609 DE-mRNAs identified in the NAFLD tissues compared with the normal tissues. Jun proto-oncogene, AP-1 transcription
\end{abstract}

Correspondence to: Dr Yuying Shen, Department of General Practice, Nanjing First Hospital, Nanjing Medical University, 68 Changle Road, Qinhuai, Nanjing, Jiangsu 210006, P.R. China E-mail: yuexiong64tunwei@163.com

\section{*Contributed equally}

Abbreviations: lncRNAs, long non-coding RNA; miRNAs, microRNA; DE-lncRNAs, differentially expressed lncRNAs; PPI, protein-protein interaction; NAFLD, non-alcoholic fatty liver disease; NASH, non-alcoholic steatohepatitis; FATP5, fatty acid transport protein 5; FDR, false discovery rate; GO, Gene Ontology; KEGG, Kyoto Encyclopedia of Genes and Genomes; CC, Closeness centrality; DC, Degree Centrality; BC, Betweenness centrality; RT-qPCR, reverse transcription quantitative polymerase chain reaction; $\mathrm{BP}$, biological process; $\mathrm{MF}$, molecular function; $\mathrm{CC}$, cellular component; HCC, hepatocellular carcinoma

Key words: non-alcoholic fatty liver disease, long non-coding RNA, mRNA, microRNA, regulatory network factor subunit (JUN), which is regulated by AC004540.4 and RP11-279F6.1, exhibited higher degree compared with other nodes in the PPI network. Furthermore, miR-409-3p and miR-139 (targeting JUN) were predicted as PPI network nodes. In the miRNA-IncRNA-mRNA network, miR-20a and B-cell lymphoma 2-like 11 (BCL2L11) were among the top 10 nodes. Additionally, BCL2L11, AC004540.4 and RP11-279F6.1 were targeted by miR-20a, miR-409-3p and miR-139 in the miRNA-IncRNA-mRNA network, respectively. RP11-279F6.1 and AC004540.4 expression was markedly enhanced in NAFLD liver tissues. These key RNAs may be involved in the pathogenic mechanisms of NAFLD.

\section{Introduction}

As the most frequently diagnosed fatty liver disease in developed countries, non-alcoholic fatty liver disease (NAFLD) occurs when fat is enriched in the liver due to non-alcoholic factors $(1,2)$. The risk factors for NAFLD include metabolic syndromes, for example combined hyperlipidemia, obesity, high blood pressure and type II diabetes mellitus and insulin resistance $(3,4)$. Non-alcoholic steatohepatitis (NASH), which is the most severe type of NAFLD, is considered to be the primary cause of cirrhosis (4). The incidence of NAFLD is 9-36.9\% globally $(5,6)$, and $20 \%$ of people in the United States of America (75-100 million people) are affected by the disease (7). Therefore, exploring the pathogenic mechanisms of NAFLD and developing novel treatment protocols are necessary.

Silencing of fatty acid transport protein 5 (FATP5) may reverse NAFLD; therefore, the activity of hepatic FATP5 is considered critical for maintaining fatty acid flux and caloric uptake during high-fat feeding (8). Patatin-like phospholipase domain containing 3 is an NAFLD-associated gene, which is closely associated with metabolic changes in hepatocytes and lipogenesis $(9,10)$. Interleukin-17 is associated with the proinflammatory response and hepatic steatosis in NAFLD and contributes to the steatosis-steatohepatitis transition (11). Several microRNAs (miRNAs), including miR-21, miR-122, miR-451 and miR-34a, are overexpressed in patients with NAFLD; in particular, miR-122 levels are associated with the grades of fatty liver disease and are a potential marker of NAFLD (12). By inhibiting the expression of 3-hydroxy-3-methylglutaryl-co-enzyme A reductase, miR-21 was demonstrated to 
mediate cholesterol and triglyceride metabolism in an NAFLD model and may be a promising diagnostic and therapeutic marker for the disease (13). miR-34a/Sirtuin 1/tumor protein p53 (p53) signaling, which is correlated with liver cell apoptosis, is inhibited by ursodeoxycholic acid and activated upon the aggravation of illness in NAFLD (14). Despite these studies, the molecular mechanisms of NAFLD are not fully understood.

In 2015, Sun et al (15) investigated the role of long non-coding RNAs (lncRNAs) in NAFLD through microarray data analysis and identified that several differentially expressed lncRNAs (DE-lncRNAs) function in the pathogenesis of NAFLD. However, the molecular regulatory mechanisms in NAFLD have not been explored in detail. Based on the expression profiles deposited by Sun et al (15), the present study additionally identified the DE-lncRNAs and differentially expressed mRNAs (DE-mRNAs) between NAFLD and normal liver tissues. In addition, key mRNAs, lncRNAs and miRNAs involved in NAFLD were also identified through protein-protein interaction (PPI) network, enrichment and miRNA-lncRNA-mRNA interaction network analyses. The expression of key RNAs were detected by reverse transcription quantitative polymerase chain reaction (RT-qPCR).

\section{Materials and methods}

Data source. The normalized expression data and annotation data from the GSE72756 dataset were acquired from the Gene Expression Omnibus (GEO) database (http://www.ncbi. nlm.nih.gov/geo/), which was generated using a GPL16956 Agilent-045997 Arraystar human lncRNA microarray V3 (Probe Name Version) platform. The sample set of GSE72756 included 5 NAFLD liver tissues (3 females and 2 males; mean age $=38.8$ years) and 5 normal liver tissues ( 3 females and 2 males; mean age $=39.2$ years). Samples used in this dataset were sourced from patients with NAFLD without other metabolic complications that were hospitalized in The Third Xiangya Hospital (Changsha, China) from March 2014 to November 2014, and NAFLD was confirmed independently by two senior pathologists by pathological examination. Liver tissues $(50-100 \mathrm{mg})$ were isolated from the patients and then rapidly frozen in liquid nitrogen. Sun et al (15) deposited the microarray dataset GSE72756, and the study was approved by the Ethics Committee of the Third Xiangya Hospital of Central South University. Informed consent was obtained from all patients.

Differential expression analysis. Based on the normalized probe expression data, the $\mathrm{R}$ package in Linear Models for Microarray Data (16) (http://www.bioconductor.org/packages/2.9/bioc/html/limma.html) was utilized to identify and annotate the differentially expressed probes between NAFLD and normal liver tissues. A false discovery rate (FDR; adjusted P-value) $<0.01$ and llog fold-change (FC) $>1$ were set as thresholds. Using the R package pheatmap (17) (http://cran.r-project. org/web/packages/pheatmap/index.html), a clustering heatmap was drawn for differentially expressed probes. According to the annotation information, the differentially expressed probes were divided into DE-IncRNAs and DE-mRNAs.

Enrichment analysis and PPInetwork analysis of DE-mRNAs. Using the R package clusterProfiler (18) (http://bioconductor.
org/packages/release/bioc/html/clusterProfiler.html), Gene Ontology (GO; http://www.geneontology.org) (19) functional and Kyoto Encyclopedia of Genes and Genomes (KEGG; http://www.genome.ad.jp/kegg) (20) pathway enrichment analyses were performed for the DE-mRNAs. The terms with FDR $<0.05$ were considered to be significant. Based on the Search Tool for the Retrieval of Interacting Genes (http://www.string-db.org/) (21) database, the interaction pairs among the DE-mRNAs were predicted with the threshold of required confidence $>0.4$. The PPI network was constructed using Cytoscape software (version 3.0.1, http://www.cytoscape.org) (22). Using the CytoNCA plugin (http://apps.cytoscape.org/apps/cytonca) (23) in Cytoscape, Closeness centrality (CC) (24), Degree Centrality (DC) (25), and Betweenness centrality (BC) (26) scores were calculated. The nodes with increased CC, DC and BC scores compared with other nodes were identified as hub nodes (27) in the PPI network.

Construction of the IncRNA-mRNA regulatory network and functional prediction of DE-lncRNAs. Based on the Pearson product-moment correlation coefficient (28), the target genes of upregulated and downregulated $\operatorname{lncRNAs}$ were predicted using a threshold of FDR $<0.05$ and correlation coefficient $>0.995$. Next, Cytoscape software (22) was utilized to draw IncRNA-mRNA regulatory networks. Additionally, enrichment analysis was conducted for the target genes of each DE-IncRNA using FDR $<0.05$ as a threshold. Using the R package clusterProfiler (18), the enriched pathways were compared to identify the significant pathways of target genes for each DE-IncRNA. An FDR $<0.05$ was used as the cut-off criterion.

Construction of miRNA-lncRNA-mRNA interaction network. Combined with the WEB-based gene set analysis toolkit (Webgestalt; http://www.webgestalt.org) (29), the miRNAs targeting DE-mRNAs involved in the PPI network were predicted. A count (number of target genes) $\geq 4$ and FDR $<0.05$ were set as the thresholds. According to the annotation information of the DE-lncRNAs, their corresponding sequences were extracted from human reference genome hg19 (30). The mature sequences of the predicted miRNAs were downloaded from the miRbase database (http://www.mirbase. org/) (31). Using MiRanda (http://www.microrna.org) (32) and RNAhybrid (http://bibiserv.techfak.uni-bielefeld.de/rnahybrid/) (33) software, IncRNAs containing significant binding sites for the aforementioned miRNAs were predicted. The predicted results of the miRanda and RNAhybrid analyses were compared to obtain the intersecting miRNA-lncRNA pairs. Based on the obtained miRNA-lncRNA and miRNA-mRNA pairs, and the associated IncRNA-mRNA and mRNA-mRNA pairs, an miRNA-lncRNA-mRNA interaction network was constructed and subjected to topological property analysis using Cytoscape software.

Sample information and RT-qPCR. A total of 2 normal liver tissues ( 2 females; age range: $47-60$ years; mean age $=53.5$ years; obtained from March to June 2017 via surgical resection) and 2 NAFLD liver tissues (2 males; age range: $41-47$ years; mean age $=44$ years; obtained from April to May 2017 via surgical 


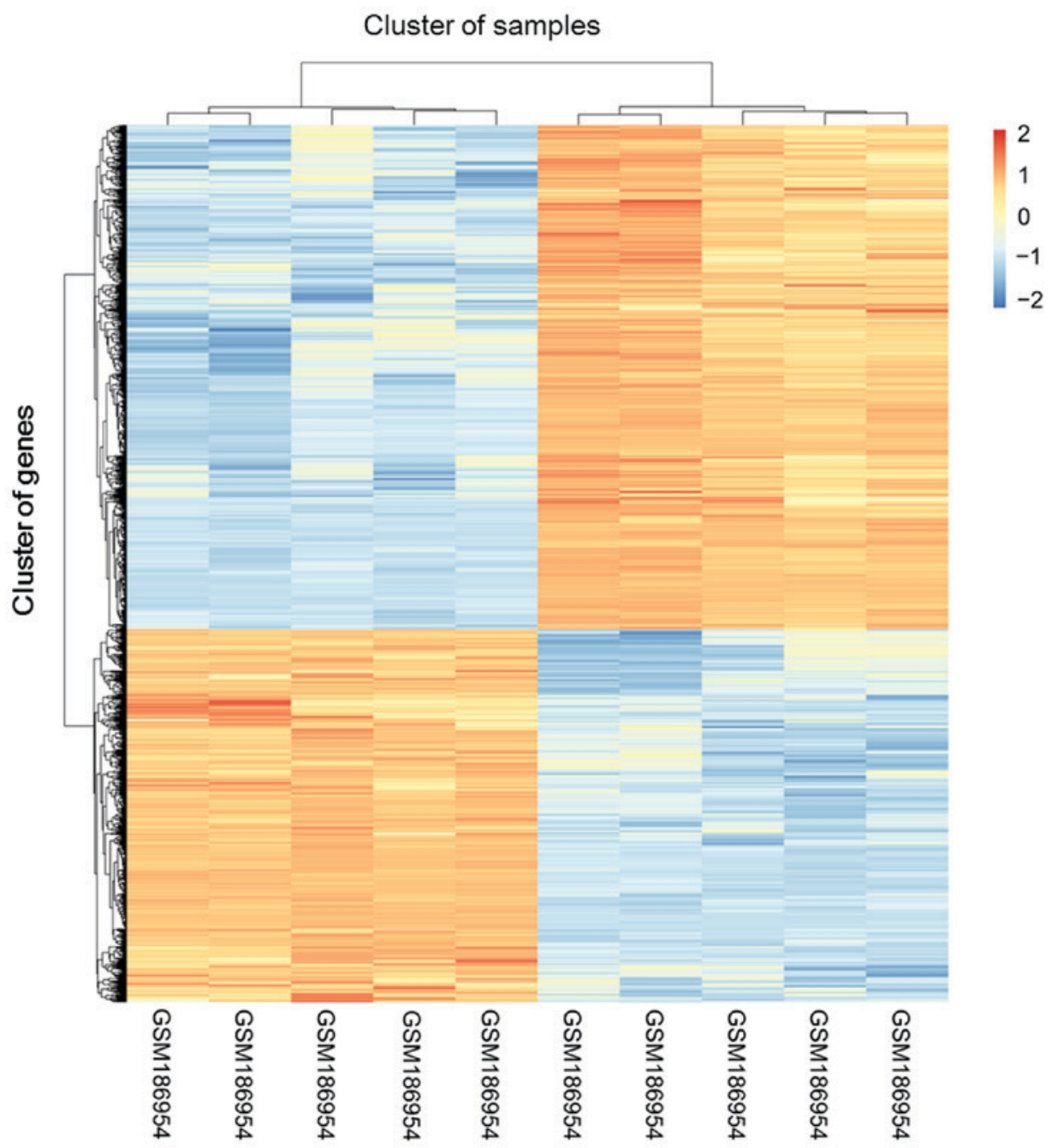

Figure 1. Clustering heatmap for the differentially expressed probes. GSM1860540, GSM1860541, GSM1860543, GSM1860545 and GSM1860548 represent normal liver tissue samples. GSM1860542, GSM1860544, GSM1860546, GSM1860547 and GSM1860549 represent non-alcoholic fatty liver disease liver tissue samples. The blue-yellow-red variation indicates alterations in expression from low to high. The y-and upper x-axes represent the cluster of genes and samples with similar alterations in expression, respectively.

resection) were provided by Nanjing First Hospital (Nanjing, China). The present study was approved by the Ethics Committee of Nanjing First Hospital. Informed consent was obtained from all patients.

Using TRIzol ${ }^{\circledR}$ reagent (Thermo Fisher Scientific, Inc., Waltham, MA, USA), total RNA was extracted from the samples. The PrimeScript RT Master MIX kit (Takara Bio, Inc., Otsu, Japan) was used to synthesize first-strand cDNA. RT-qPCR was performed using Power SYBR Green PCR Master Mix (cat. no., 4367659; Thermo Fisher Scientific, Inc.) on an ABI 7500 FAST real-time PCR system (Applied Biosystems; Thermo Fisher Scientific, Inc.). The thermocycler conditions for qPCR were as follows: Initial denaturation $\left(50^{\circ} \mathrm{C}, 3 \mathrm{~min}\right) ; 40$ cycles of denaturation $\left(95^{\circ} \mathrm{C}\right.$, $3 \mathrm{~min})$, annealing $\left(95^{\circ} \mathrm{C}, 10 \mathrm{sec}\right)$, and extension $\left(60^{\circ} \mathrm{C}, 30 \mathrm{sec}\right)$. The specificity of the primer amplicons was examined by melting curve analysis. The comparative $\mathrm{Cq}$ method (34) was employed to quantify target mRNA and miRNA expression. mRNA expression was normalized to that of GAPDH. The primers used in the present study were as follows: GAPDH-forward (F)-5'-TGACAACTTTGGTATCGTGGA
AGG-3'; GAPDH-reverse (R)-5'-AGGCAGGGATGATGT TCTGGAGAG-3'; RP11-279F6.1-h-F-5'-CGGACATAGCCA ACGCACCT-3'; RP11-279F6.1-R-5'-TTCATACTTCTGCTG CGTCCA-3'; AC004540.4-F-5'-TTCACAACACACTCAAAG CCT-3'; AC004540.4-R-5'-CAACTGCACTCCAAATGG CTA-3'.

Statistical analysis. All data are expressed as the mean \pm standard error of the mean, and the differences between the two groups were compared by Student's t-test. Statistical analyses were performed using SPSS 22.0 software (IBM Corp., Armonk, NY, USA), and GraphPad Prism 5 (GraphPad Software, Inc., La Jolla, CA, USA) was used to visualize the results. $\mathrm{P}<0.05$ was considered to indicate a statistically significant difference.

\section{Results}

Differential expression analysis. The clustering heatmap for differentially expressed probes is presented in the Fig. 1. There were 318 DE-lncRNAs, including 105 upregulated and 213 downregulated, and 609 DE-mRNAs, including 
Table I. Top 5 functions and pathways enriched in the analysis of differentially expressed mRNAs.

\begin{tabular}{|c|c|c|c|c|c|c|}
\hline Category & Term & ID & Description & P-value & FDR & Count \\
\hline \multirow[t]{20}{*}{ Upregulated } & GO_BP & GO:0019752 & Carboxylic acid metabolic process & $2.90 \times 10^{-24}$ & $1.24 \times 10^{-20}$ & 69 \\
\hline & GO_BP & GO:0032787 & $\begin{array}{l}\text { Monocarboxylic acid metabolic } \\
\text { process }\end{array}$ & $7.56 \times 10^{-24}$ & $1.61 \times 10^{-20}$ & 53 \\
\hline & GO_BP & GO:0006082 & Organic acid metabolic process & $1.14 \times 10^{-23}$ & $1.62 \times 10^{-20}$ & 73 \\
\hline & GO_BP & GO:0043436 & Oxoacid metabolic process & $2.13 \times 10^{-23}$ & $2.27 \times 10^{-20}$ & 72 \\
\hline & GO_BP & GO:0044281 & Small molecule metabolic process & $7.36 \times 10^{-22}$ & $6.27 \times 10^{-19}$ & 110 \\
\hline & GO_CC & GO:0044444 & Cytoplasmic part & $1.98 \times 10^{-14}$ & $8.66 \times 10^{-12}$ & 206 \\
\hline & GO_CC & GO:0044432 & Endoplasmic reticulum part & $1.50 \times 10^{-12}$ & $3.27 \times 10^{-10}$ & 55 \\
\hline & GO_CC & GO:0005783 & Endoplasmic reticulum & $3.69 \times 10^{-12}$ & $5.38 \times 10^{-10}$ & 68 \\
\hline & GO_CC & GO:0044421 & Extracellular region part & $5.42 \times 10^{-12}$ & $5.93 \times 10^{-10}$ & 120 \\
\hline & GO_CC & GO:0005789 & Endoplasmic reticulum membrane & $1.20 \times 10^{-11}$ & $1.05 \times 10^{-9}$ & 48 \\
\hline & GO_MF & GO:0003674 & Molecular function & $2.54 \times 10^{-17}$ & $1.73 \times 10^{-14}$ & 324 \\
\hline & GO_MF & GO:0003824 & Catalytic activity & $1.18 \times 10^{-15}$ & $4.01 \times 10^{-13}$ & 163 \\
\hline & GO_MF & GO:0048037 & Cofactor binding & $4.10 \times 10^{-12}$ & $9.30 \times 10^{-10}$ & 25 \\
\hline & GO_MF & GO:0016491 & Oxidoreductase activity & $8.89 \times 10^{-12}$ & $1.51 \times 10^{-9}$ & 41 \\
\hline & GO_MF & GO:0050662 & Coenzyme binding & $2.34 \times 10^{-8}$ & $3.18 \times 10^{-6}$ & 17 \\
\hline & KEGG Pathway & hsa01100 & Metabolic pathways & $1.02 \times 10^{-11}$ & $2.65 \times 10^{-9}$ & 72 \\
\hline & KEGG Pathway & hsa00040 & $\begin{array}{l}\text { Pentose and glucuronate } \\
\text { interconversions }\end{array}$ & $2.24 \times 10^{-5}$ & $2.59 \times 10^{-3}$ & 7 \\
\hline & KEGG Pathway & hsa01200 & Carbon metabolism & $3.94 \times 10^{-5}$ & $2.59 \times 10^{-3}$ & 12 \\
\hline & KEGG Pathway & hsa00830 & Retinol metabolism & $4.32 \times 10^{-5}$ & $2.59 \times 10^{-3}$ & 9 \\
\hline & KEGG Pathway & hsa05204 & Chemical carcinogenesis & $4.99 \times 10^{-5}$ & $2.59 \times 10^{-3}$ & 10 \\
\hline \multirow[t]{17}{*}{ Downregulated } & GO_BP & GO:0044699 & Single-organism process & $4.23 \times 10^{-16}$ & $1.52 \times 10^{-12}$ & 197 \\
\hline & GO_BP & GO:0032501 & Multicellular organismal process & $1.48 \times 10^{-12}$ & $1.85 \times 10^{-9}$ & 128 \\
\hline & GO_BP & GO:0044707 & Single-multicellular organism process & $1.54 \times 10^{-12}$ & $1.85 \times 10^{-9}$ & 125 \\
\hline & GO_BP & GO:0008150 & Biological process & $6.60 \times 10^{-12}$ & $5.93 \times 10^{-9}$ & 210 \\
\hline & GO_BP & GO:0051128 & $\begin{array}{l}\text { Regulation of cellular component } \\
\text { organization }\end{array}$ & $1.24 \times 10^{-11}$ & $8.92 \times 10^{-9}$ & 58 \\
\hline & GO_CC & GO:0005615 & Extracellular space & $1.54 \times 10^{-13}$ & $5.95 \times 10^{-11}$ & 52 \\
\hline & GO_CC & GO:0044449 & Contractile fiber part & $1.04 \times 10^{-11}$ & $2.02 \times 10^{-9}$ & 19 \\
\hline & GO_CC & GO:0005576 & Extracellular region & $2.82 \times 10^{-11}$ & $3.64 \times 10^{-9}$ & 103 \\
\hline & GO_CC & GO:0043292 & Contractile fiber & $4.73 \times 10^{-11}$ & $4.58 \times 10^{-9}$ & 19 \\
\hline & GO_CC & GO:0030017 & Sarcomere & $1.73 \times 10^{-10}$ & $1.34 \times 10^{-8}$ & 17 \\
\hline & GO_MF & GO:0003674 & Molecular function & $1.33 \times 10^{-11}$ & $6.89 \times 10^{-9}$ & 213 \\
\hline & GO_MF & GO:0005515 & Protein binding & $8.48 \times 10^{-9}$ & $2.19 \times 10^{-6}$ & 159 \\
\hline & GO_MF & GO:0008307 & Structural constituent of muscle & $1.73 \times 10^{-8}$ & $2.99 \times 10^{-6}$ & 8 \\
\hline & GO_MF & GO:0008092 & Cytoskeletal protein binding & $3.61 \times 10^{-8}$ & $4.67 \times 10^{-6}$ & 29 \\
\hline & GO_MF & GO:0005488 & Binding & $1.29 \times 10^{-6}$ & $1.34 \times 10^{-6}$ & 187 \\
\hline & Pathway & hsa04261 & $\begin{array}{l}\text { Adrenergic signaling in } \\
\text { cardiomyocytes }\end{array}$ & $2.73 \times 10^{-4}$ & $4.34 \times 10^{0}$ & 8 \\
\hline & Pathway & hsa00512 & Mucin type O-glycan biosynthesis & $4.43 \times 10^{-4}$ & $4.34 \times 10^{0}$ & 4 \\
\hline
\end{tabular}

GO, Gene Ontology; BP, biological process; CC, cell component; MF, molecular function; KEGG, Kyoto Encyclopedia of Genes and Genomes; FDR, false discovery rate.

353 upregulated and 256 downregulated, in the NAFLD liver tissues compared with the normal liver tissues.

Enrichment analysis and PPI network analysis of DE-mRNAs. The top $5 \mathrm{GO}$ 'biological process' (BP), GO 'cellular component' (CC), GO 'molecular function' (MF) and KEGG terms enriched in the DE-mRNAs analysis are summarized in Table I. For the upregulated mRNAs, the enriched GO and KEGG terms primarily included 'carboxylic acid metabolic process' (GO_BP; FDR $=1.24 \times 10^{-20}$ ), 


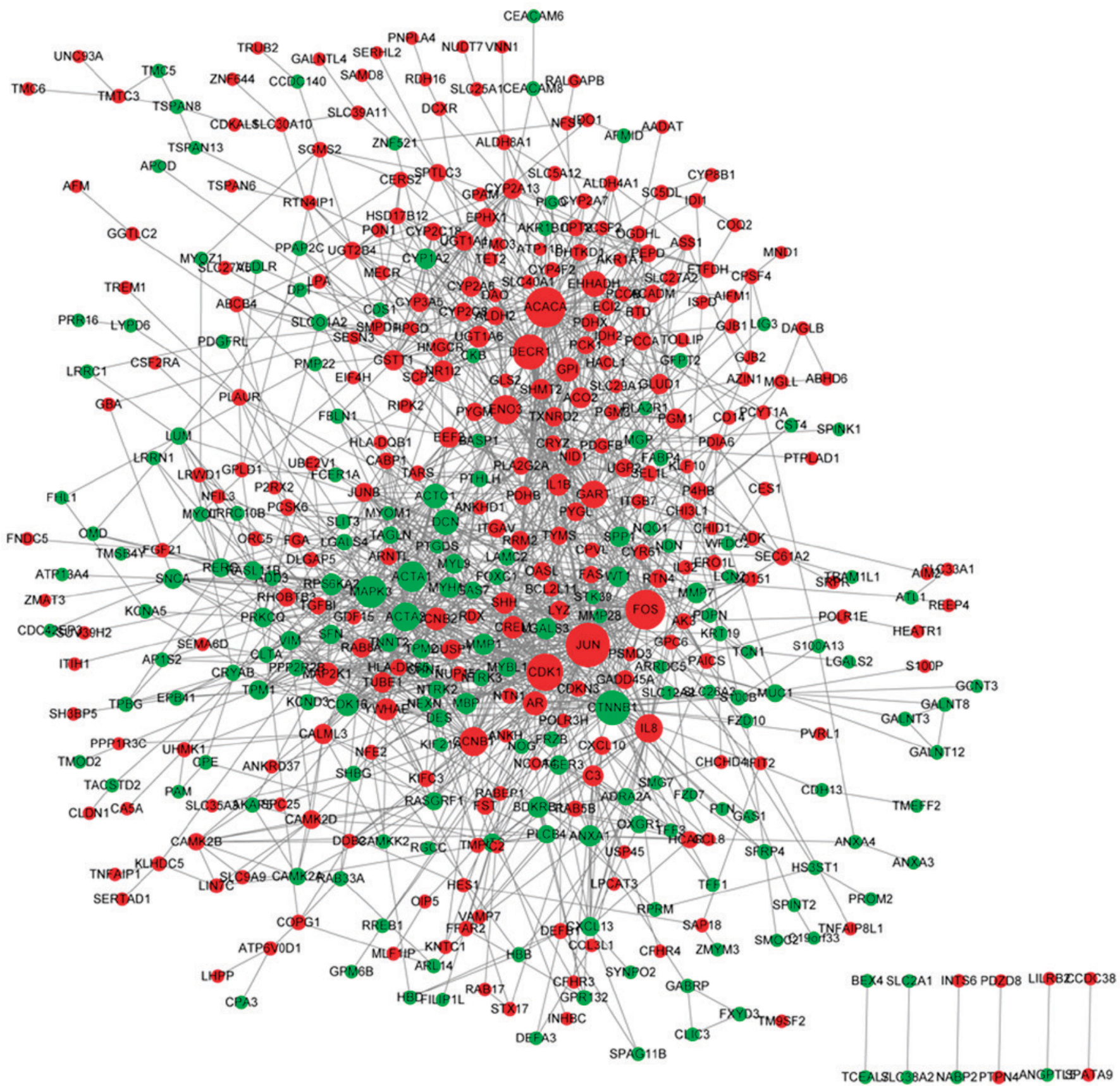

Figure 2. Protein-protein interaction network of differentially expressed mRNAs. Red and green dots represent upregulated and downregulated mRNAs, respectively. Node size represents the degree of the node.

'cytoplasmic part' (GO_CC; FDR=8.66x10-12), 'molecular

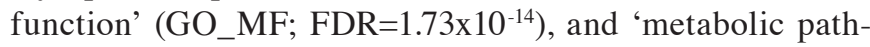

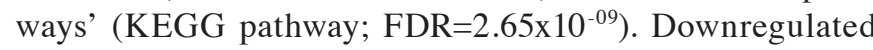
mRNAs were primarily enriched in 'single-organism process' (GO_BP; FDR=1.52 $\times 10^{-12}$ ), 'extracellular space'

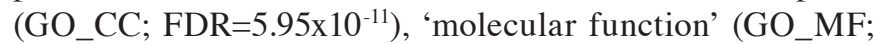
$\mathrm{FDR}=6.89 \times 10^{-09}$ ), and 'adrenergic signaling in cardiomyocytes' (KEGG pathway; FDR $=4.34 \times 10^{-2}$ ). The PPI network for the DE-mRNAs involved 442 nodes and 1,409 edges, and it was identified that Jun proto-oncogene, AP-1 transcription factor subunit (JUN) interacted with B-cell lymphoma 2 (Bcl-2)-like 11 (BCL2L11), as demonstrated in Fig. 2. The top 15 nodes, including JUN, with the highest BC, CC and DC scores are summarized in Table II.
Construction of the lncRNA-mRNA regulatory network and functional prediction of DE-lncRNAs. Following prediction of the target genes of the upregulated and downregulated IncRNAs, IncRNA-mRNA regulatory networks were constructed. For the upregulated IncRNAs, the lncRNA-mRNA regulatory network contained 182 nodes (including 37 lncRNAs and 145 target genes) and 672 interactions. For the downregulated lncRNAs, the IncRNA-mRNA regulatory network contained 140 nodes (including 47 lncRNAs and 93 target genes) and 450 interactions, among which AC004540.4 and RP11-279F6.1 were identified to target JUN. The top 10 nodes (including AC004540.4 and RP11-279F6.1) in the 1ncRNA-mRNA regulatory networks are summarized in Table III. Subsequent to enrichment 
Table II. Top 15 nodes with increased DC, BC and CC scores in the protein-protein interaction network.

\begin{tabular}{|c|c|c|c|c|c|}
\hline \multicolumn{2}{|c|}{ DC } & \multicolumn{2}{|c|}{$\mathrm{BC}$} & \multicolumn{2}{|c|}{$\mathrm{CC}$} \\
\hline mRNA & Score & mRNA & Score & mRNA & Score \\
\hline JUN & 59 & JUN & 33383.63 & JUN & 0.069956 \\
\hline ACACA & 52 & ACACA & 23963.01 & FOS & 0.069231 \\
\hline FOS & 51 & CTNNB1 & 18141.12 & CTNNB1 & 0.069133 \\
\hline CDK1 & 45 & FOS & 16604.96 & DECR1 & 0.068756 \\
\hline DECR1 & 42 & DECR1 & 15936.45 & MAPK3 & 0.068542 \\
\hline CTNNB1 & 41 & CDK1 & 12244.42 & ACACA & 0.068531 \\
\hline MAPK3 & 36 & IL8 & 11466.93 & ACTA1 & 0.068425 \\
\hline ACTA1 & 32 & MAPK3 & 10861.69 & ACTA2 & 0.068383 \\
\hline ENO3 & 31 & ACTA1 & 9482.17 & CREM & 0.068351 \\
\hline CCNB1 & 31 & GART & 8161.233 & CDK1 & 0.068277 \\
\hline IL8 & 29 & MBP & 7587.735 & $\mathrm{AR}$ & 0.068087 \\
\hline ACTA2 & 29 & $\mathrm{AR}$ & 6690.792 & GART & 0.068024 \\
\hline GART & 28 & ENO3 & 6230.197 & IL8 & 0.068014 \\
\hline EHHADH & 24 & DCN & 6073.046 & CCNB1 & 0.067804 \\
\hline DCN & 23 & RTN4IP1 & 5927.582 & ENO3 & 0.067784 \\
\hline
\end{tabular}

BC, Betweenness centrality; CC, Closeness centrality; DC, Degree Centrality.

Table III. Top 10 nodes in the lncRNA-mRNA regulatory networks for the upregulated and downregulated lncRNAs.

\begin{tabular}{lcllc}
\hline \multicolumn{2}{c}{ Upregulated } & & & \multicolumn{2}{c}{ Downregulated } \\
\cline { 1 - 2 } \cline { 5 - 5 } Symbol & Degree & & \multicolumn{1}{c}{ Symbol } & Degree \\
\hline AC004540.4 & 60 & & XLOC_014103 & 47 \\
RP11-279F6.1 & 54 & & C17orf76-AS1 & 45 \\
AQP7P1 & 53 & & RP11-279F6.3 & 41 \\
XLOC_007896 & 52 & & AK027145 & 41 \\
AK025288 & 51 & & RP11-13L2.4 & 34 \\
RP1-60O19.1 & 47 & & RP11-120K9.2 & 30 \\
TRHDE-AS1 & 46 & & LOC100505806 & 29 \\
RP11-345M22.2 & 42 & & BC073897 & 26 \\
LOC100422737 & 38 & & RP11-471J12.1 & 21 \\
AK055386 & 29 & & RMST & 14 \\
\hline
\end{tabular}

lncRNA, long non-coding RNA.

analysis, the enriched pathways for the target genes of each upregulated and downregulated IncRNA were compared to identify significant pathways.

Construction of miRNA-lncRNA-mRNA interaction network. The miRNAs (including miR-409-3p and miR-139, which targeted JUN) targeting the DE-mRNAs involved in the PPI network are summarized in Table IV. Subsequent to prediction of the binding sites between DE-lncRNAs and the miRNAs associated with the miRNA-mRNA pairs, an miRNA-IncRNA interaction network was constructed (involving 26 miRNAs, 111 lncRNAs and 224 interactions). In the miRNA-lncRNA interaction network, miR-409-3p and miR-139 interacted with AC004540.4 and RP11-279F6.1, respectively. The top 10 nodes exhibiting the highest degrees are summarized in Table V. Finally, an miRNA-lncRNA-mRNA interaction network was constructed, which contained 249 nodes, including 36 miRNAs, 95 lncRNAs, 118 mRNAs and 845 interactions (Fig. 3). The top 10 nodes [including miR-20a; solute carrier family 30, member 10, (SLC30A10); and BCL2L11] with the highest degrees in the miRNA-lncRNA-mRNA interaction network are summarized in Table VI. In particular, BCL2L11 was targeted by miR-20a in the interaction network.

RP11-279F6.1 and AC004540.4 expression. The RT-qPCR results revealed that RP11-279F6.1 and AC004540.4 expression levels were markedly enhanced in the liver tissues from patients with NAFLD compared with the control liver samples (Fig. 4; $\mathrm{P}<0.01$ ).

\section{Discussion}

In the present study, 318 DE-lncRNAs, including 105 upregulated and 213 downregulated lncRNAs, and 609 DE-mRNAs, including 353 upregulated and 256 downregulated mRNAs, were screened in the NAFLD liver tissues compared with normal liver tissues. In the PPI network for DE-mRNAs, JUN, the targeting gene of BCL2L11, was among the top 15 nodes. JUN was targeted by the lncRNAs RP11-279F6.1 and AC004540.4 and miRNAs miR-409-3p and miR-139. Additionally, miR-409-3p and miR-139 were predicted as the DE-mRNAs involved in the PPI network. Additionally, miR-409-3p and miR-139 were regulated 
Table IV. miRNAs targeting the differentially expressed mRNAs involved in the protein-protein interaction network.

\begin{tabular}{|c|c|c|}
\hline miRNA & Gene count & P-value \\
\hline hsa_CAGTATT, miR-200B, miR-200C, miR-429 & 19 & $\operatorname{adjP}=0.00004$ \\
\hline$h s a \_A C C A A A G, m i R-9$ & 16 & $\operatorname{adjP}=0.00220$ \\
\hline hsa_AACATTC, miR-409-3P & 8 & $\operatorname{adj} \mathrm{P}=0.00220$ \\
\hline hsa_GGCAGCT, miR-22 & 10 & $\operatorname{adjP}=0.00220$ \\
\hline hsa_ACTGTAG,miR-139 & 7 & $\operatorname{adj} \mathrm{P}=0.00240$ \\
\hline hsa_ACATTCC, miR-1, miR-206 & 11 & $\operatorname{adjP}=0.00240$ \\
\hline hsa_AAAGGAT, miR-501 & 7 & $\operatorname{adjP}=0.00240$ \\
\hline hsa_TGTTTAC, miR-30A-5P, miR-30C, & 16 & $\operatorname{adjP}=0.00240$ \\
\hline \multicolumn{3}{|l|}{$m i R-30 D, m i R-30 B, m i R-30 E-5 P$} \\
\hline hsa_GTGCCTT,miR-506 & 19 & $\operatorname{adj} \mathrm{P}=0.00240$ \\
\hline$h s a \_T A C T T G A, m i R-26 A, m i R-26 B$ & 11 & $\operatorname{adj} \mathrm{P}=0.00240$ \\
\hline$h s a \_A C T T T A T, m i R-142-5 P$ & 11 & $\operatorname{adjP}=0.00240$ \\
\hline hsa_GTGCCAT, miR-183 & 8 & $\operatorname{adjP}=0.00360$ \\
\hline hsa_TGGTGCT,miR-29A, miR-29B, miR-29C & 14 & $\operatorname{adjP}=0.00670$ \\
\hline hsa_ACTGTGA, miR-27A, miR-27B & 13 & $\operatorname{adjP}=0.00680$ \\
\hline hsa_GCACTTT, miR-17-5P, miR-20A, miR-106A, & 15 & $\operatorname{adjP}=0.00700$ \\
\hline \multicolumn{3}{|l|}{$m i R-106 B, m i R-20 B, m i R-519 D$} \\
\hline hsa_GTGCAAT, miR-25, miR-32, miR-92, miR-363, miR-367 & 10 & $\operatorname{adjP}=0.00760$ \\
\hline hsa_TCTGATC, miR-383 & 4 & $\operatorname{adjP}=0.00820$ \\
\hline hsa_CCTGTGA, miR-513 & 6 & $\operatorname{adjP}=0.00920$ \\
\hline
\end{tabular}

hsa, Homo sapiens; miR, microRNA; adjP, adjusted P-value.

Table V.Top 10 nodes with highest degrees in the miRNA-lncRNA interaction network.

\begin{tabular}{lcccc}
\hline \multicolumn{2}{c}{ miRNA } & & & \multicolumn{2}{c}{ lncRNA } \\
\cline { 1 - 2 } \cline { 5 - 5 } Symbol & Degree & & Symbol & Degree \\
\hline hsa-miR-92a & 32 & & C17orf76-AS1 & 9 \\
hsa-miR-367 & 21 & & AC079776.2 & 8 \\
hsa-miR-20b & 21 & & RP4-669L17.4 & 6 \\
hsa-miR-17-5p & 21 & & XLOC_001223 & 5 \\
hsa-miR-145 & 18 & & XLOC_000638 & 5 \\
hsa-miR-25 & 15 & & RP11-365O16.3 & 5 \\
hsa-miR-20a & 15 & & RP11-315I20.1 & 5 \\
hsa-miR-106a & 11 & & RP11-261C10.3 & 5 \\
hsa-miR-501 & 10 & & RP11-249C24.3 & 5 \\
hsa-miR-106b & 9 & & BC005927 & 5 \\
\hline
\end{tabular}

hsa, Homo sapiens; miRNA/miR, microRNA; lncRNA, long non-coding RNA.

by AC004540.4 and RP11-279F6.1, respectively. In the miRNA-1ncRNA-mRNA interaction network, miR-20a, SLC30A10 and BCL2L11 were among the top 10 nodes. RP11-279F6.1 and AC004540.4 expression was markedly increased in the NAFLD patient liver tissues compared with the control liver samples.
JUN, also known as $A P 1$, was identified to be increased in a previous study of NAFLD (35). Phosphorylation of the transcriptional activation domain of $A P 1$ is conducted by JNKs to enhance its activity, thereby accelerating the progression and development of NASH $(36,37)$. JUN is considered to be an oncogene in the liver, and its expression is enhanced in response to inflammatory stimuli, promoting liver tumorigenesis $(38,39)$. JUN serves an important role in hepatitis B virus-associated tumorigenesis by promoting the proliferation of hepatocytes and dysplasia progression, indicating that JUN is a useful treatment target for preventing hepatitis-associated tumorigenesis (40). The results from the present study indicated that JUN is involved in the pathogenesis of NAFLD.

In the present study, JUN interacted with BCL2L11 and was targeted by miR-20a in the miRNA-lncRNA-mRNA interaction network. The serum/plasma level of miR-20a has potential value for detecting hepatitis $\mathrm{C}$ virus (HCV) infection, and therefore circulating miR-20a may be useful as a predictive marker in liver fibrosis mediated by HCV (41). Apoptosis is a major cause of hepatocyte elimination in NAFLD, and inhibition of the anti-apoptotic protein $\mathrm{Bcl}-2$ and activation of the pro-apoptotic protein p53 promotes inflammation in NAFLD (42). Overexpression of Bcl-2 results in resistance to reperfusion injury in the ischemic liver by suppressing apoptosis and is associated with increased caspase 3 and cytoplasmic cytochrome $c$ and a deficiency of Bcl-extra large (43). By targeting the anti-apoptotic gene $\mathrm{Bcl}-2$, miR-15b and miR-16 regulate tumor necrosis factor-mediated hepatic apoptosis in the process of acute liver failure (44). The data 


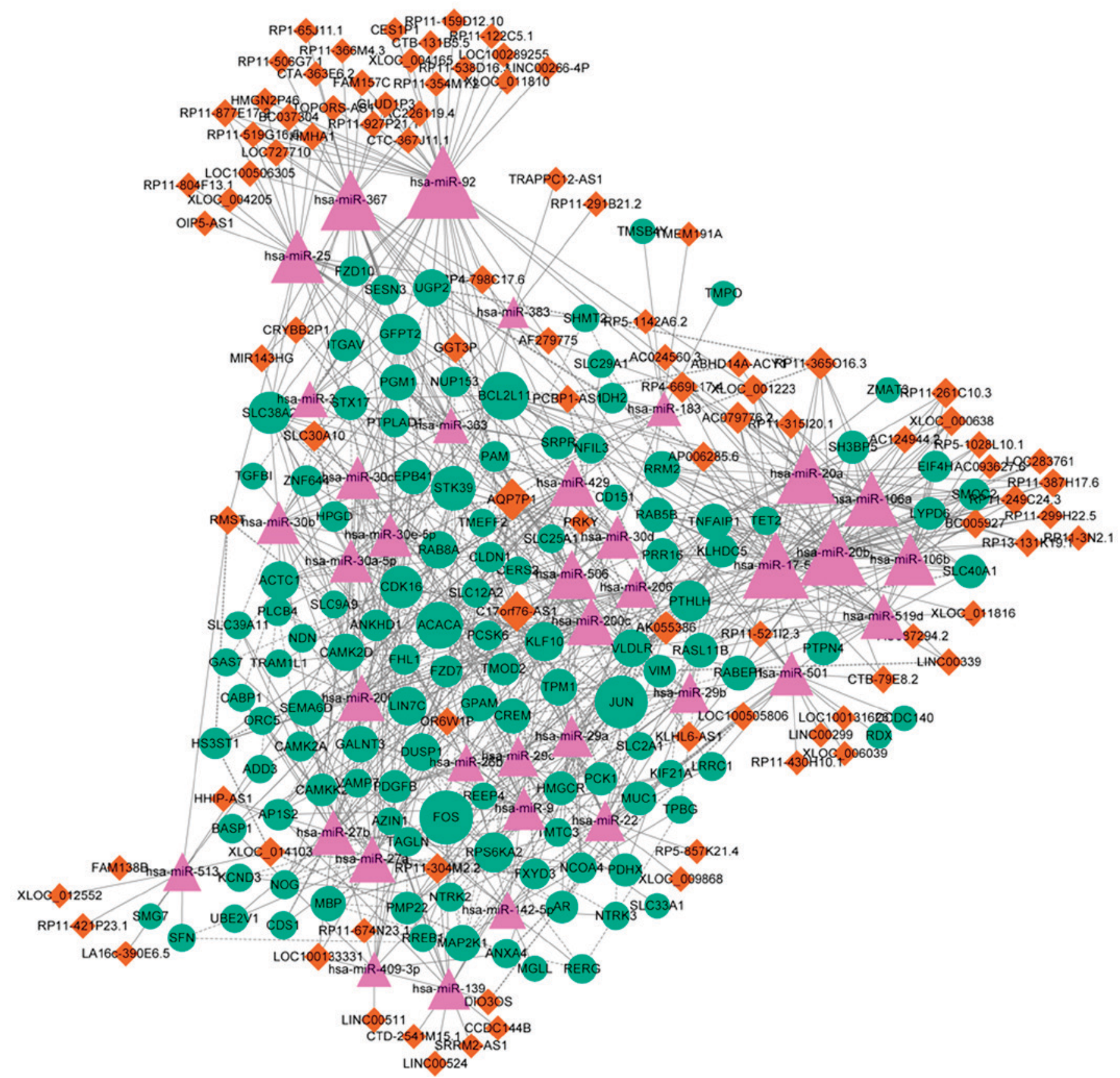

Figure 3. miRNA-lncRNA-mRNA interaction network. Orange diamonds, pink triangles and green circles represent lncRNAs, miRNAs and mRNAs, respectively. Node size represents the degree of the node. IncRNA, long non-coding RNA; miRNA, microRNA.
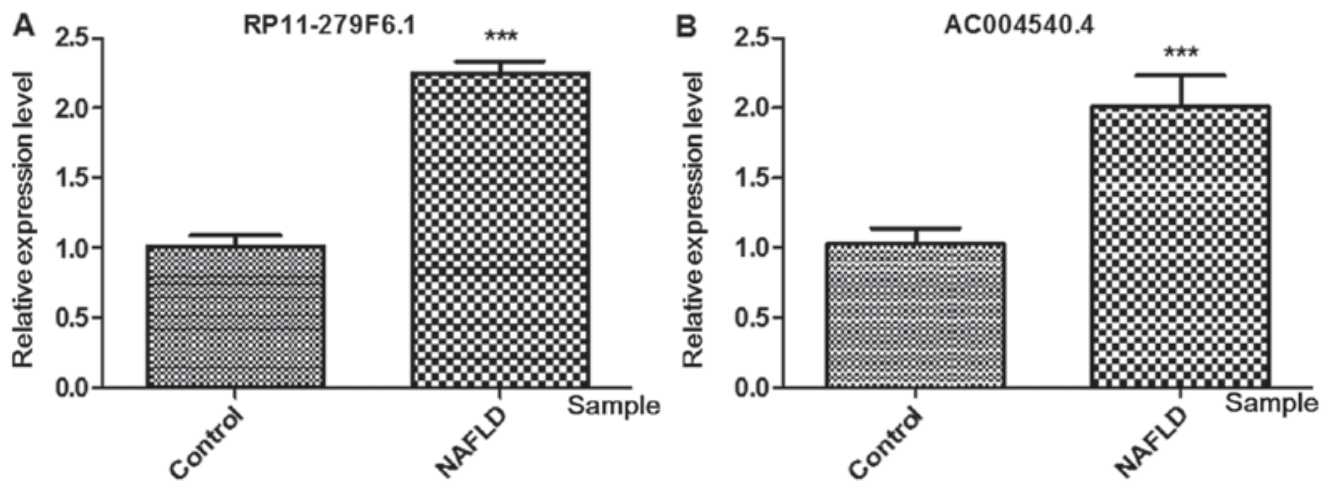

Figure 4. Expression levels of (A) RP11-279F6.1 and (B) AC004540.4 in the control and NAFLD liver tissues by reverse transcription quantitative polymerase chain reaction. ${ }^{* * *} \mathrm{P}<0.01$ vs. control. NAFLD, non-alcoholic fatty liver disease. 
Table VI. Top 10 nodes with highest degrees in the miRNA-lncRNA-mRNA interaction network.

\begin{tabular}{|c|c|c|c|c|c|}
\hline \multicolumn{2}{|c|}{ miRNA } & \multicolumn{2}{|c|}{ lncRNA } & \multicolumn{2}{|c|}{ mRNA } \\
\hline Symbol & Degree & Symbol & Degree & Symbol & Degree \\
\hline hsa-miR-92 & 42 & $A Q P 7 P 1$ & 15 & FOS & 25 \\
\hline$h s a-m i R-20 b$ & 36 & C17orf76-AS1 & 14 & $J U N$ & 24 \\
\hline hsa-miR-17-5p & 36 & AK055386 & 8 & BCL2L11 & 20 \\
\hline hsa-miR-367 & 31 & RP11-365016.3 & 7 & $A C A C A$ & 19 \\
\hline$h s a-m i R-20 a$ & 30 & AC079776.2 & 7 & STK39 & 17 \\
\hline hsa-miR-106a & 26 & SLC $30 A 10$ & 6 & SLC $38 A 2$ & 15 \\
\hline hsa-miR-25 & 25 & RP4-669L17.4 & 6 & $C D K 16$ & 15 \\
\hline hsa-miR-106b & 24 & XLOC_014103 & 5 & GFPT2 & 14 \\
\hline hsa-miR-506 & 21 & XLOC_000638 & 5 & PTHLH & 14 \\
\hline$h s a-m i R-200 c$ & 20 & XLOC_001223 & 5 & RPS6KA2 & 13 \\
\hline
\end{tabular}

Hsa, Homo sapiens; miRNA/miR, microRNA; lncRNA, long non-coding RNA.

from the present study suggest that miR-20a serves a role in NAFLD by targeting BCL2L11 and affecting the expression of JUN.

JUN was also regulated by the miRNAs of miR-409-3p and miR-139. In hepatoma HuH-7 cells, miR-409-3p decreased the production of fibrinogen by downregulating fibrinogen beta chain precursor expression (45). A previous study suggested that miR-409-3p may be utilized to detect the progression of NAFLD (46). miR-409-3p was also identified as a biomarker for the therapeutics and diagnosis of a number of heart failure-associated diseases and a risk factor of NAFLD (47). Overexpression of miR-139, which was downregulated in hepatocellular carcinoma (HCC) samples, suppresses the progression and metastasis of $\mathrm{HCC}$ by downregulating rho-kinase 2 , indicating that miR-139 may be used to predict the outcome of HCC (48). In the present study, miR-409-3p and miR-139 interacted with the lncRNAs of AC004540.4 and RP11-279F6.1, respectively. Furthermore, the expression levels of AC004540.4 and RP11-279F6.1 were markedly enhanced in liver tissues from patients with NAFLD compared with the control liver samples. Therefore, we hypothesized that miR-409-3p, miR-139, AC004540.4 and RP11-279F6.1 co-regulated JUN expression in patients with NAFLD.

Although the present study succeeded in identifying specific key miRNAs and lncRNAs in the development of NAFLD, there were also certain limitations. For example, the analyses were based on the microarray dataset GSE72756 downloaded from the GEO database. However, the stages of NAFLD (fatty liver, steatohepatitis or fibrosis/cirrhosis) were not clearly described in the GEO database. Therefore, the degree of NAFLD was not clear. In addition, the sample size was too small, and the predicted regulatory associations were not validated. Future studies will aim to confirm the predicted regulatory associations using cell line experiments.

In conclusion, 318 DE-lncRNAs and 609 DE-mRNAs were identified in NAFLD liver tissues by bioinformatics analysis. Additionally, specific mRNAs (including JUN and BCL2L11) and miRNAs (including miR-20a, miR-409-3p and miR-139) may serve essential roles in the pathogenesis of
NAFLD. The lncRNAs AC004540.4 and RP11-279F6.1 were also implicated in the mechanisms of NAFLD.

\section{Acknowledgements}

Not applicable.

\section{Funding}

No funding was received.

\section{Availability of data and materials}

The datasets used and/or analyzed during the current study are available from the corresponding author on reasonable request.

\section{Authors' contributions}

HW and XS were responsible for the conception and design of the research and drafting the manuscript. ZT and YL performed the data acquisition. JZ performed the data analysis and interpretation. YS participated in the design of the study and performed the statistical analysis. All authors have read and approved the manuscript.

\section{Ethics approval and consent to participate}

The present study was approved by the Ethics Committee of Nanjing First Hospital. Informed consent was obtained from all patients.

\section{Patient consent for publication}

Informed consent was obtained from all patients.

\section{Competing interests}

The authors declare that they have no competing interests. 


\section{References}

1. Shaker M, Tabbaa A, Albeldawi M and Alkhouri N: Liver transplantation for nonalcoholic fatty liver disease: New challenges and new opportunities. World J Gastroenterol 20: 5320-5330, 2014.

2. Rinella ME: Nonalcoholic fatty liver disease: A systematic review. JAMA 313: 2263, 2015.

3. Tolman KG and Dalpiaz AS: Treatment of non-alcoholic fatty liver disease. Ther Clin Risk Manag 3: 1153-1163, 2007.

4. Clark JM and Diehl AM: Nonalcoholic fatty liver disease: An underrecognized cause of cryptogenic cirrhosis. JAMA 289: 3000-3004, 2003.

5. Omagari K, Kadokawa Y, Masuda J, Egawa I, Sawa T, Hazama H, Ohba K, Isomoto H, Mizuta Y, Hayashida K, et al: Fatty liver in non-alcoholic non-overweight Japanese adults: Incidence and clinical characteristics. J Gastroenterol Hepatol 17: 1098-1105, 2002.

6. Shen L, Fan JG, Shao Y, Zeng MD, Wang JR, Luo GH, Li JQ and Chen SY: Prevalence of nonalcoholic fatty liver among administrative officers in Shanghai: An epidemiological survey. World J Gastroenterol 9: 1106-1110, 2003.

7. Lazo M, Hernaez R, Bonekamp S, Kamel IR, Brancati FL, Guallar E and Clark JM: Non-alcoholic fatty liver disease and mortality among US adults: Prospective cohort study. BMJ 343: d6891, 2011.

8. Doege H, Grimm D, Falcon A, Tsang B, Storm TA, Xu H, Ortegon AM, Kazantzis M, Kay MA and Stahl A: Silencing of hepatic fatty acid transporter protein 5 in vivo reverses diet-induced non-alcoholic fatty liver disease and improves hyperglycemia. J Biol Chem 283: 22186-22192, 2008.

9. Hoekstra M, Li ZS, Kruijt JK, Van Eck M, Van Berkel TJ and Kuiper J: The expression level of non-alcoholic fatty liver disease-related gene PNPLA3 in hepatocytes is highly influenced by hepatic lipid status. J Hepatol 52: 244-251, 2010

10. Lin YC, Chang PF, Hu FC, Yang WS, Chang MH and Ni YH: A common variant in the PNPLA3 gene is a risk factor for non-alcoholic fatty liver disease in obese Taiwanese children J Pediatr 158: 740-744, 2010.

11. Tang Y, Bian Z, Zhao L, Liu Y, Liang S, Wang Q, Han X, Peng Y, Chen X, Shen L, et al: Interleukin-17 exacerbates hepatic steatosis and inflammation in non-alcoholic fatty liver disease. Clin Exp Immunol 166: 281-290, 2011.

12. Yamada H, Suzuki K, Ichino N, Ando Y, Sawada A, Osakabe K, Sugimoto K, Ohashi K, Teradaira R, Inoue T, et al: Associations between circulating microRNAs (miR-21, miR-34a, miR-122 and miR-451) and non-alcoholic fatty liver. Clin Chim Acta 424 99-103, 2013.

13. Sun C, Huang F, Liu X, Xiao X, Yang M, Hu G, Liu H and Liao L: miR-21 regulates triglyceride and cholesterol metabolism in non-alcoholic fatty liver disease by targeting HMGCR. Int J Mol Med 35: 847-853, 2015.

14. Rui EC, Ferreira DM, Afonso MB, Borralho PM, Machado MV, Cortez-Pinto $\mathrm{H}$ and Rodrigues CM: miR-34a/SIRT1/p53 is suppressed by ursodeoxycholic acid in the rat liver and activated by disease severity in human non-alcoholic fatty liver disease. J Hepatol 58: 119-125, 2012.

15. Sun C, Liu X, Yi Z, Xiao X, Yang M, Hu G, Liu H, Liao L and Huang F: Genome-wide analysis of long noncoding RNA expression profiles in patients with non-alcoholic fatty liver disease. IUBMB Life 67: 847-852, 2015.

16. Smyth GK: Limma: Linear models for microarray data. In: Bioinformatics and Computational Biology Solutions Using R and Bioconductor. Gentleman R, Carey VJ, Huber W, Irizarry RA and Dudoit S, (eds). Springer New York, New York, NY, pp397-420, 2005

17. Kolde R and Kolde MR: Package 'pheatmap'. https://cran.r-project. org/web/packages/pheatmap/. Accessed October 12, 2015.

18. Yu G, Wang LG, Han Y and He QY: clusterProfiler: An $\mathrm{R}$ package for comparing biological themes among gene clusters. OMICS 16: 284-287, 2012

19. Tweedie S, Ashburner M, Falls K, Leyland P, McQuilton P, Marygold S, Millburn G, Osumi-Sutherland D, Schroeder A, Seal R, et al: FlyBase: Enhancing drosophila gene ontology annotations. Nucleic Acids Res 37 (Database Issue): D555-D559, 2009.

20. Altermann E and Klaenhammer TR: PathwayVoyager: Pathway mapping using the Kyoto encyclopedia of genes and genomes (KEGG) database. BMC Genomics 6: 60, 2005.
21. Szklarczyk D, Franceschini A, Wyder S, Forslund K, Heller D, Huerta-Cepas J, Simonovic M, Roth A, Santos A, Tsafou KP, et al: STRING v10: Protein-protein interaction networks, integrated over the tree of life. Nucleic Acids Res 43 (Database Issue): D447-D452, 2015.

22. Saito R, Smoot ME, Ono K, Ruscheinski J, Wang PL, Lotia S, Pico AR, Bader GD and Ideker T: A travel guide to Cytoscape plugins. Nat Methods 9: 1069-1076, 2012.

23. Tang Y, Li M, Wang J, Pan Y and Wu FX: CytoNCA: A cytoscape plugin for centrality analysis and evaluation of biological networks. Biosystems 127: 67-72, 2015.

24. Du Y, Gao C, Chen X, Hu Y, Sadiq R and Deng Y: A new closeness centrality measure via effective distance in complex networks. Chaos 25: 033112, 2015.

25. Opsahl T, Agneessens F and Skvoretz J: Node centrality in weighted networks: Generalizing degree and shortest paths. Social Networks 32: 245-251, 2010.

26. Cukierski WJ and Foran DJ: Using betweenness centrality to identify manifold Shortcuts. Proc IEEE Int Conf Data Min 2008: 949-958, 2008

27. He $\mathrm{X}$ and Zhang $\mathrm{J}$ : Why do hubs tend to be essential in protein networks? PLoS Genet 2: e88, 2006

28. Mukaka MM: Statistics corner: A guide to appropriate use of correlation coefficient in medical research. Malawi Med J 24: 69-71, 2012

29. Wang J, Duncan D, Shi Z and Zhang B: WEB-based GEne SeT AnaLysis Toolkit (WebGestalt): Update 2013. Nucleic Acids Res 41: W77-W83, 2013

30. Kent WJ, Sugnet CW, Furey TS, Roskin KM, Pringle TH, Zahler AM and Haussler D: The human genome browser at UCSC. Genome Res 12: 996-1006, 2002.

31. Kozomara A and Griffiths-Jones S: miRBase: Annotating high confidence microRNAs using deep sequencing data. Nucleic Acids Res 42 (Database Issue): D68-D73, 2014.

32. John B, Enright AJ, Aravin A, Tuschl T, Sander C and Marks DS: Correction: Human MicroRNA targets. PLoS Biol 3: e264, 2005.

33. Krüger J and Rehmsmeier M: RNAhybrid: microRNA target prediction easy, fast and flexible. Nucleic Acids Res 34: W451-W454, 2006.

34. Livak KJ and Schmittgen TD: Analysis of relative gene expression data using real-time quantitative PCR and the 2(-Delta Delta C(T)) method. Methods 25: 402-408, 2001.

35. Dorn C, Engelmann JC, Saugspier M, Koch A, Hartmann A, Müller M, Spang R, Bosserhoff A and Hellerbrand C: Increased expression of c-Jun in nonalcoholic fatty liver disease. Lab Invest 94: 394-408, 2014

36. Singh R, Wang YJ, Xiang YQ, Tanaka KE, Gaarde WA and Czaja MJ: Differential effects of JNK1 and JNK2 inhibition on murine steatohepatitis and insulin resistance. Hepatology 49: 87-96, 2009.

37. Karin M and Gallagher E: From JNK to pay dirt: Jun kinases, their biochemistry, physiology and clinical importance. IUBMB Life 57: 283-295, 2005.

38. Min L, Ji Y, Bakiri L, Qiu Z, Cen J, Chen X, Chen L, Scheuch H, Zheng H, Qin L, et al: Liver cancer initiation is controlled by AP-1 through SIRT6-dependent inhibition of survivin. Nat Cell Biol 15: 1203-1211, 2013 .

39. Machida K, Tsukamoto H, Liu JC, Han YP, Govindarajan S, Lai MM, Akira S and Ou JH: c-Jun mediates hepatitis $\mathrm{C}$ virus hepatocarcinogenesis through signal transducer and activator of transcription 3 and nitric oxide-dependent impairment of oxidative DNA repair. Hepatology 52: 480-492, 2010.

40. Trierweiler C, Hockenjos B, Zatloukal K, Thimme R, Blum HE, Wagner EF and Hasselblatt $\mathrm{P}$ : The transcription factor c-JUN/AP-1 promotes HBV-related liver tumorigenesis in mice. Cell Death Differ 23: 576-582, 2016.

41. Shrivastava S, Petrone J, Steele R, Lauer GM, Di Bisceglie AM and Ray RB: Up-regulation of circulating miR-20a is correlated with hepatitis $\mathrm{C}$ virus-mediated liver disease progression. Hepatology 58: 863-871, 2013.

42. Panasiuk A, Dzieciol J, Panasiuk B and Prokopowicz D: Expression of p53, Bax and Bcl-2 proteins in hepatocytes in non-alcoholic fatty liver disease. World J Gastroenterol 12: 6198-6202, 2006.

43. Selzner M, Rüdiger HA, Selzner N, Thomas DW, Sindram D and Clavien PA: Transgenic mice overexpressing human B cl-2 are resistant to hepatic ischemia and reperfusion. J Hepatol 36: 218-225, 2002. 
44. An F, Gong B, Wang H, Yu D, Zhao G, Lin L, Tang W, Yu H, Bao $S$ and Xie Q: miR-15b and miR-16 regulate TNF mediated hepatocyte apoptosis via BCL2 in acute liver failure. Apoptosis 17: 702-716, 2012.

45. Fort A, Borel C, Migliavacca E, Antonarakis SE, Fish RJ and Neerman-Arbez M: Regulation of fibrinogen production by microRNAs. Blood 116: 2608-2615, 2010.

46. Tryndyak VP, Marrone AK, Latendresse JR, Muskhelishvili L, Beland FA and Pogribny IP: MicroRNA changes, activation of progenitor cells and severity of liver injury in mice induced by choline and folate deficiency. J Nutr Biochem 28: 83-90, 2016.

47. Yang Y, Yu T, Jiang S, Zhang Y, Li M, Tang N, Ponnusamy M, Wang JX and Li PF: miRNAs as potential therapeutic targets and diagnostic biomarkers for cardiovascular disease with a particular focus on WO2010091204. Expert Opin Ther Pat 27: 1021-1029, 2017
48. Wong CC, Wong CM, Tung EK, Au SL, Lee JM, Poon RT, Man K and Ng IO: The microRNA miR-139 suppresses metastasis and progression of hepatocellular carcinoma by down-regulating Rho-kinase 2. Gastroenterology 140: 322-331, 2011.

(i) () $($ This work is licensed under a Creative Commons Attribution-NonCommercial-NoDerivatives 4.0 International (CC BY-NC-ND 4.0) License. 